\title{
Micromechanical Response of Fibre-Reinforced Materials using the Boundary Element Technique.
}

\author{
M. G. KNIGHT ${ }^{\dagger *}$, L. C. WROBEL ${ }^{*}$ and J. L. HENSHALL ${ }^{*}$ \\ ${ }^{*}$ Department of Mechanical Engineering, Brunel University, Uxbridge, UK. UB8 3PH. \\ " Department of Mechanical \& Manufacturing Engineering, Nottingham Trent University, Nottingham, \\ UK. NG1 4BU. \\ † Corresponding author: matthew.knight@brunel.ac.uk
}

\begin{abstract}
The Boundary Element Method (BEM) and the Embedded Cell Approach (ECA) have been used to analyse the effects of constituent material properties and fibre spatial distribution on the localised behaviour of a transversely loaded, unidirectional fibre-reinforced composite. The geometrical structures examined were perfectly periodic, uniformly spaced fibre arrangements in square and hexagonal embedded cells and ten cells in which 60 fibres were randomly placed within the matrix. The models involve both elastic fibres and matrix, with the interfaces between the different phases being fully bonded. The results indicate that both the fibre packing and the material properties of the constituent phases have a significant effect on the overall stress distribution and the magnitude of localised stress concentrations within a composite. Non-periodic arrangements give rise to higher local stresses, and the magnitudes of these stress concentrations have a strong dependence on the ligament length (distance between the two neighbouring fibres that have a common high-stress region), and to a lesser extent on the angle relative to the applied load (angle between a plane containing the two fibre centres and the applied load). Furthermore, analysis of a three-phase composite, comprised of a mixture of both stiff and compliant fibres, had higher stress concentrations than the equivalent two-phase composites.
\end{abstract}

Keywords: boundary element method, multi-phase materials, embedded cell approach, periodic and random arrangements. 


\section{INTRODUCTION}

Many industrial and engineering materials, as well as the majority of 'natural' materials, are inhomogeneous, i.e. they consist of dissimilar constituents (or 'phases') that are distinguishable at some length scale(s). Each constituent shows different material properties and/or material orientations, and may again be inhomogeneous at some smaller length scale. Some typical representatives of inhomogeneous materials are composites, polycrystalline materials, porous and cellular materials, functionally graded materials, wood, and bone. However, within this paper, only structural composite materials will be considered. The advantage of composite materials is that, if well designed, they usually exhibit the best qualities of their constituents and often some qualities that neither constituent possesses. Some of the properties that can be improved by forming a composite material include stiffness, strength, wear resistance, and fatigue life.

Because of the inherently heterogeneous nature of composite materials, they are conveniently studied from two points of view: macromechanics and micromechanics. Macromechanics is the study of material behaviour wherein the material is presumed homogeneous, and the effects of constituent materials are detected only as averaged apparent macroscopic properties. In the present paper, macromechanics will not be considered, hence for a more formal treatment of the underlying concepts see e.g. Jones, 1999.

The term micromechanics implies the study of material behaviour wherein the interaction of the constituent phases is examined on a microscopic scale to determine their effect on the properties of the composite. There are many theoretical micromechanics models that have been developed and applied to predict the effective properties of composite materials in terms of size, shape, volume fraction and distribution of the constituent phases. In particular, predictions of elastic properties such as Young's modulus, shear modulus, and the Poisson's ratio of two-phase materials have been studied extensively, and comparisons with experimental data have been 
widely examined (Hashin, 1962; Hashin and Shtrikman, 1963; Hashin and Rosen, 1964; Budiansky, 1965; Hill, 1965; Walpole, 1966; Foye, 1966; Adams and Doner, 1967; Halpin and Tsai, 1967; Mori and Tanaka, 1973; Willis, 1977; Christensen, 1990).

Although these analytical models are attractive, because they offer better physical insight, any attempt to extend them to complex geometries invariably leads to mathematical intractability. However, in recent years, the growing accessibility of fast computers has allowed the development of advanced computational methods to replace the time-consuming analytical procedures. Among the various numerical methods available, the most popular are the Finite Difference Method (FDM), Finite Element Method (FEM) and the Boundary Element Method (BEM).

In this study, an in-house developed BEM program has been implemented to analyse the transverse tensile response of a 'composite construct'. A composite construct refers to a simplified model that closely represents the local behaviour within a real composite material. In the present paper, a unidirectional composite is simplified to a two-dimensional model representing the geometrical characteristics within an arbitrary cross-sectional plane perpendicular to the fibre axes, where the fibres are assumed to be equally sized and circular, and either distributed periodically or randomly within the embedding matrix. The BEM is particularly well suited for this type of problem, since variations in the geometrical detail can be considered with only very minor adaptations of the elements being required. By comparison with the FEM, it also has the advantage that, due to the higher order of accuracy of the computed stresses, a lesser degree of mesh refinement is needed to obtain similar accuracies. This is important within the present study, as high stress concentrations would be expected in regions of near-touching inclusions (Knight et al., 2002).

The literature describing this powerful technique has grown in recent years (see Brebbia et al., 1984; and Becker, 1992; and, more recently, Wrobel and Aliabadi, 2002). Hence, only a brief explanation of the technique relevant to the present analyses will be presented. 
The boundary, surface or interface, is divided into a number of segments, each of the segments being known as an 'element', and the whole assembly of elements being known as the 'mesh'. Over these discretised boundaries the transformed governing differential equations, in the form of integral identities, are then numerically integrated. Therefore, provided that the boundary conditions are satisfied, a system of linear algebraic equations emerges, for which a unique solution can be obtained.

Many of the early numerical models of composite materials were restricted to a unit-cell approach, in which the real structure is approximated by a periodic array of certain regular geometries. For example, Eischen and Torquato (1993) applied the BEM to a simplified unit-cell, for the determination of the effective elastic moduli of a fibrous composite material. An idealised hexagonal array of infinitely long fibres was considered together with a variety of inclusions, which were either stiffer or weaker than the matrix; and a wide range of volume fractions of fibres were examined. Chati and Mitra (1998) then extended this work to account for transversely isotropic materials and the effects of crenulations and interfacial debonding of the fibres. In most cases, the results from these and other similar numerical models compare well with experimental data. However, as with particulate composites, no single approach has been universally recognised to simply and accurately model the elastic response of real fibre-reinforced composites, for all practical situations.

The purpose of the current work is to apply a numerical tool to a parametric investigation of the micromechanics of composite materials, and thus provide an improved knowledge of the influence of fibre arrangement and phase properties on the local stresses. A range of geometrical parameters and materials properties has been modelled with a view to designing composite materials with improved properties that are more closely optimised for the particular specification required.

The present paper describes the micromechanical response within a transversely loaded, unidirectionally reinforced composite, where the effects of fibre distribution and constituent material properties are parametrically examined. The fibres are assumed to 
be circular and equal in area, the matrix and fibres follow an elastic response, and the interface between the fibres and the matrix is fully bonded. The numerical simulations include several simple geometric arrangements, i.e. square and hexagonally arranged fibres. These models are then extended to accommodate random distributions, so that the effects of fibre clustering on the localised stress distributions could be investigated. In addition, a range of material combinations, i.e. relatively stiff or flexible fibres or a mixture of both, relative to the matrix, is considered. An embedded cell approach (ECA) is employed to apply far field loading, as discussed within the following section, and a statistical method, based on the Weibull distribution, has been used to describe the distribution of stress concentrations.

\section{MODEL DESCRIPTION}

\subsection{EMBEDDED CELL APPROACH (ECA)}

The embedded cell approach is a combination of macro- and micromechanics modelling, which aims at predicting the microfields in inhomogeneous materials at high spatial resolution. This technique approximates the real inhomogeneous material by a model consisting of a core containing a discrete phase arrangement ('local heterogeneous region'), which is embedded within some outer region to which far field loads or displacements are applied. A schematic depiction of the technique can be seen in Fig. 1. The material characteristics of the inner core can range from relatively simple idealised configurations to highly detailed experimentally obtained phase arrangements. The outer region may be described by some macroscopic constitutive law (Wulf et al., 1996; and Monaghan and Brazil, 1997), determined self-consistently from the behaviour of the core (Chen et al., 1994; and Dong and Schmauder, 1996), or it may take the form of a coarse description and/or discretisation of the global phase arrangement (Sautter et al., 1993). 


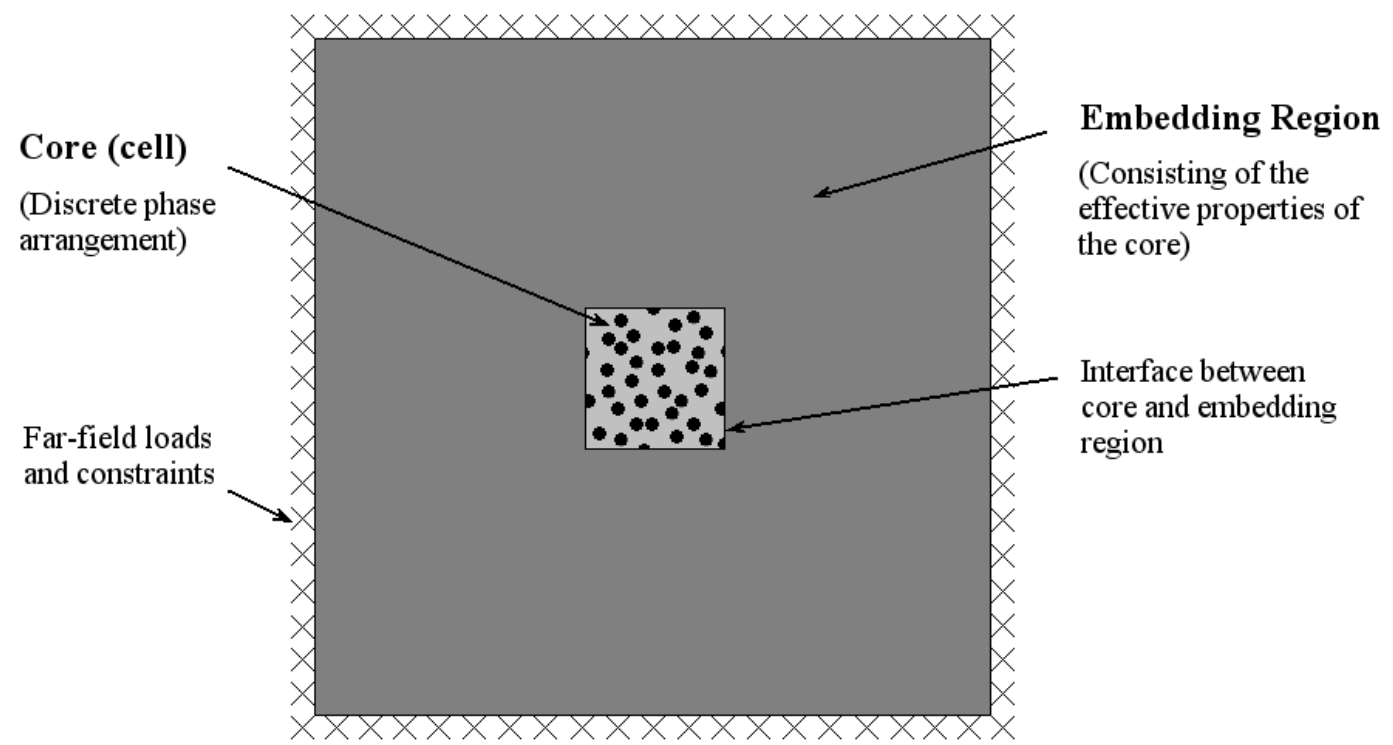

Figure 1 Concept of the Embedded Cell Approach (ECA).

ECAs have been successfully used for materials characterisation, and they are also very well suited for studying regions of special interest, e.g. local evolution of damage around a crack tip (Wulf et al., 1996). However, a suitable description of the outer region must be chosen, so that errors in the accommodation of stresses and strains are avoided, and some care is required with respect to spurious 'boundary layers' that may occur at the interface between the core and the surrounding material. Note that these boundary layers are a consequence of the modelling approach only, and do not have any physical background. Typically, they have a thickness of about an inclusion diameter for elastic materials, but they may be longer ranged for nonlinear material behaviour (Böhm, 2000).

\subsection{GEOMETRY AND MATERIALS}

The distribution of the unidirectional fibres within a matrix is modelled using several different packing arrangements, which can be classified into two groups: (i) embedded cells containing regularly spaced periodic fibre arrangements and (ii) embedded cells containing randomly distributed fibres. For the embedded cells with uniformly spaced fibre arrangements, the fibres were packed in square or hexagonal arrays as shown in 
Fig. 2. These two idealised distributions are the most common arrangements used in many previous ‘unit-cell’ analyses (Foye, 1966; Adams and Doner, 1967; Aboudi, 1989; Guild and Young, 1989; Achenbach and Zhu, 1989).

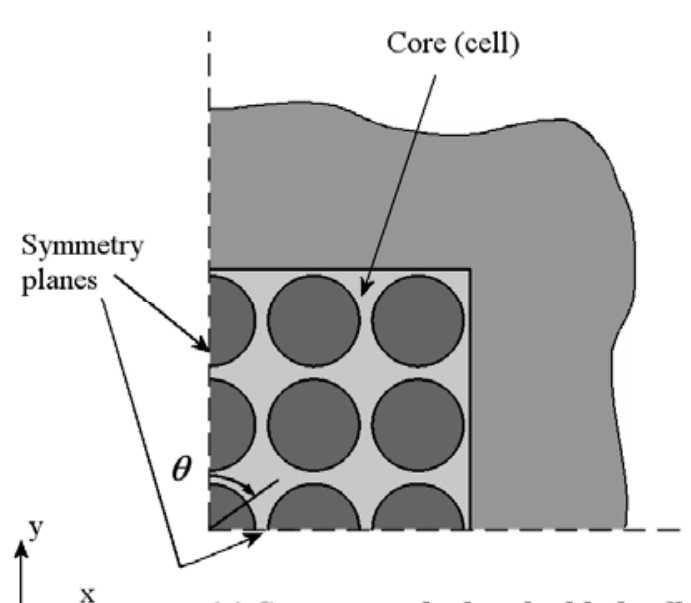

(a) Square-packed embedded cell

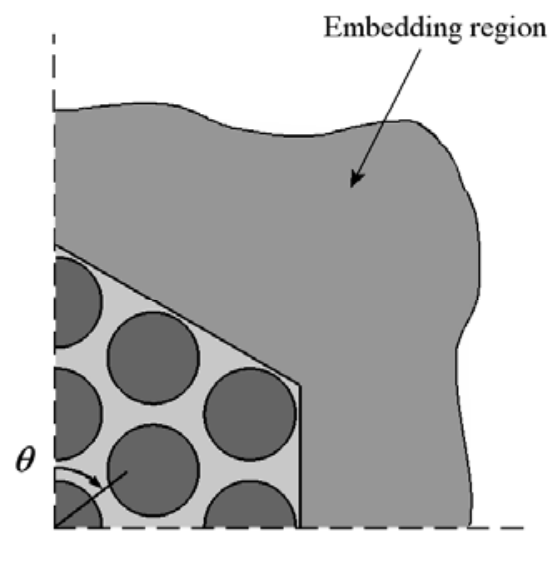

(b) Hexagonal-packed embedded cell

Figure 2 Schematic of regularly spaced periodic fibre packing arrangements. (a) 25 square packed fibres, and (b) 19 hexagonally packed fibres.

For the square (SQ) and hexagonal (HEX) periodic arrangements, as shown in Fig. 2(a) and 2(b), respectively, only one-quarter of the problem needs to be modelled, due to the symmetry. Along these symmetry planes the BE model need not be discretised and symmetry boundary conditions are applied. In the particular models shown, 25 and 19 equally sized fibres form the square and hexagonal lattices, respectively. However, as discussed in section 3.1.1, further models of differing numbers of inclusions are analysed to quantify the 'boundary layer' produced by the ECA. In each case, the embedding region is subjected to a remote unit tensile stress $\left(\sigma_{o}\right)$ in the $y$ direction, at a sufficient distance from the core such that free surface effects can be neglected.

In addition to the uniformly spaced fibre arrangements in Fig. 2, numerical simulations were conducted using embedded cells containing 60 equally sized, randomly (RAND) distributed fibres, as shown in Fig. 3. These random-fibre embedded cells were generated using an in-house developed program. Within this program, the stipulated 
fibre volume fraction and number of inclusions determines the size of the embedded cell, and the location of each fibre is randomly selected such that fibres neither overlap with each other nor with the cell boundaries. Note that by restricting fibres from overlapping with the cell boundaries, modelling the problem is greatly simplified. However, the localised fibre volume fraction close to the cell walls becomes lower than that of the central region (or kernel), as shown later. The effect of this geometric characterisation is discussed in section 3.2.1. Furthermore, the ligament size (distance between the fibres) is controlled such that numerical problems could be avoided, as discussed in section 2.3. Although the number of fibres chosen does not guarantee that the sample size accurately represents the response of a real composite, previous studies have confirmed that the representative volume element (RVE) selected is sufficient in size to adequately capture the behaviour of an inhomogeneous material (Nakamura and Suresh, 1993; Drugan and Willis, 1996; Gusev, 1997).

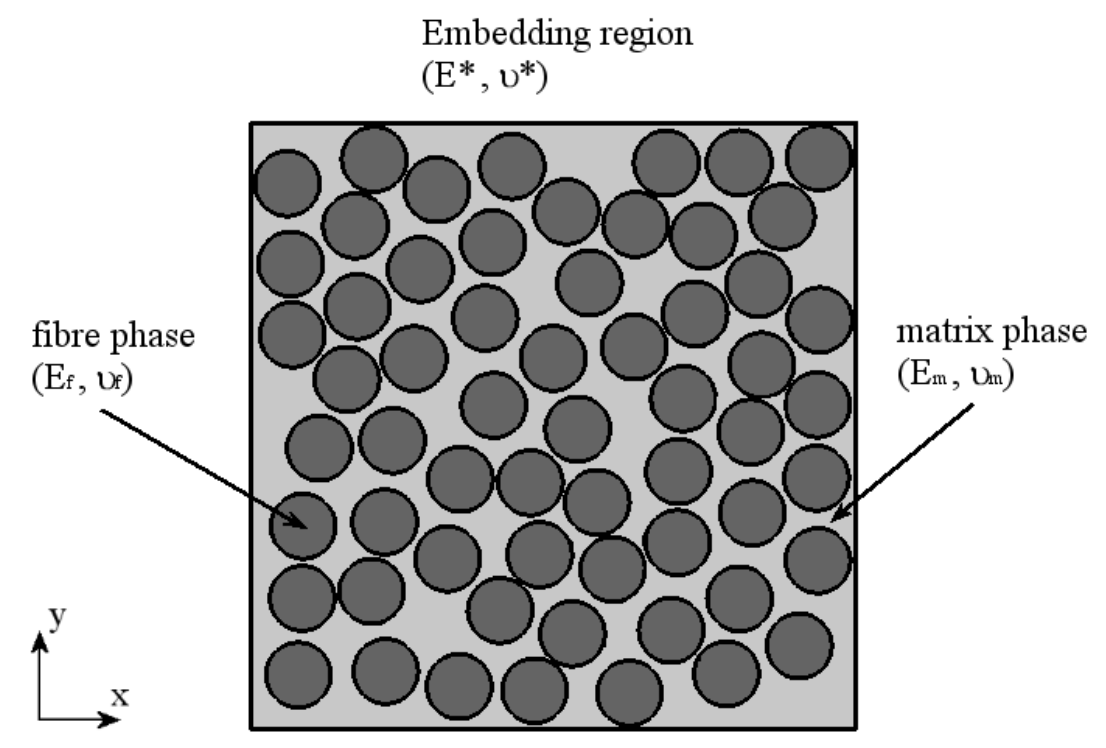

Figure 3 Schematic representation of an embedded cell containing 60 randomly distributed fibres. 
In this study, ten different randomly distributed embedded cells are analysed so that a sample of results could be used within a statistical analysis, without increasing the model size and computational resources required. In addition, by conducting multiple simulations, a greater insight into the effect of random inclusion dispersion could then be achieved.

Representative material properties were chosen for the analysis, such that the results may be examined parametrically. Hence, the overall trends presented are equally valid for any fibre-reinforced composite with similar materials and interfacial characteristics. The volume fraction of fibres $\left(V_{f}\right)$ used within the numerical models is $0.2,0.4$ and 0.6 for the periodic arrangements, and 0.54 (which corresponds to a kernel fibre volume fraction of approximately 0.6, as shown in section 3.2.1) for the random arrangements. These values were deemed to cover the scope of typical reinforcement concentrations for fibre-reinforced composites, with the emphasis on the higher concentration $\left(V_{f}=0.6\right)$ used within both the periodic and random models.

For the initial models considered, the Young's modulus ratio selected for the two phases is $E_{f} / E_{m}=10$, and the Poisson's ratio $v_{f}=v_{m}=0.3$, where the subscript $f=$ fibres and $m=$ matrix. Further studies then consider the effect of varying the Young's moduli ratio $\left(E_{f} / E_{m}\right)$, and Poisson's ratio mismatch $\left(v_{f} / v_{m}\right)$ on the localised stress concentrations. The cases considered were; the fibres are nearly rigid $\left(E_{f} / E_{m}=100\right)$, the fibres are compliant $\left(E_{f} / E_{m}=0.1\right)$, the fibres have a higher Poisson's ratio than the matrix ( $v_{f}=$ $\left.0.4, v_{m}=0.2\right)$, and finally the fibres have a lower Poisson's ratio than the matrix $\left(v_{f}=\right.$ $\left.0.2, v_{m}=0.4\right)$. In the first two models the Poisson's ratio of each constituent is 0.3, whereas, the latter models have an elastic moduli ratio of 10.

Finally, composite models comprising mixtures of both stiff and compliant fibres relative to that of the matrix were analysed. This has direct relevance to the recent development of improved fracture toughness composites that contain mixtures of both high and low Young's moduli particles (Hornsby and Premphet, 1997,1998). Considering an idealised composite, which contains particles whose Young's modulus 
is greater than the matrix, it would generally be expected that the overall strength would increase, but inadvertently the toughness generally decreases. In contrast, composites containing particles whose Young's modulus is less than the matrix may be significantly tougher, but they are also generally relatively weak. Therefore, by manufacturing a material with a dispersion of high and low Young's moduli inclusions, both strength and toughness could possibly be enhanced, due to the combined reinforcing and toughening effects of the three-phase system. The material properties chosen were a 50:50 mixture of stiff fibres $\left(E_{f} / E_{m}=10\right)$, and compliant fibres $\left(E_{f} / E_{m}=0.1\right)$, with each constituent having a Poisson's ratio of 0.3. Ten arrangements were analysed, whose geometric characteristics were kept constant, however the distribution of fibre property was randomly varied.

For all the models considered, the material properties $\left(E^{*}, v^{*}\right)$ employed for the embedding region were obtained using semi-empirical expressions proposed by Halpin and Tsai (1967), as follows:

$$
M^{*}=M_{m} \frac{1+\xi \eta V_{f}}{1-\eta V_{f}}
$$

where

$$
\eta=\frac{M_{f}-M_{m}}{M_{f}+\xi M_{m}}
$$

in which:

$M=$ Material property $\left(E_{2}, G_{12}, v_{12}\right)$

$V_{f}=$ Fibre-volume fraction

$\xi=$ Adjustable parameter (depends on the fibre geometry, packing geometry, and loading conditions)

Although the given expressions only form an approximate representation of more complicated micromechanics models, they in general give good agreement with experiment over the complete range of fibre content (Wolfenden and Wolla, 1989). The only difficulty in using the Halpin-Tsai equations is in the determination of a suitable value of $\xi$. For the present study, $\xi=2$ was used in the transverse material calculations, as it was found to give excellent agreement with Foye’s (1966) results for fibres with 
square cross sections in a diamond array, and Adams and Doner's (1967) results for circular fibres in a square array.

\subsection{BOUNDARY ELEMENT ANALYSIS}

In this study, an in-house developed BEM program has been used to examine the different facets of the research. This BEM code is suitable for two-dimensional (plane strain and plane stress) problems, and allows the use of linear and quadratic boundary elements. An iterative solver, namely the generalized minimal residual (GMRES) method (Saad and Schultz, 1986), is implemented within the code to substantially reduce the computational time to solve large systems of equations. For instance, a problem involving 8000 degrees of freedom (dof) solved on a typical desktop computer took in the region of six hours using $L U$ decomposition (direct method), compared to approximately forty minutes using GMRES, for similar accuracies.

The BEM meshes for the plane strain models (which are equivalent to the inclusions representing fibres) have been carefully developed, such that finer elements are placed along the fibre/matrix interfaces in regions where the fibres are nearly touching. This is to ensure accurate results where possible high stress gradients occur. Although rigorous studies of mesh refinement were not carried out for all the models, due to the high computational cost, we are confident that all the results presented in this study pertain to boundary element meshes which are sufficiently fine to provide mesh-independent results. Typical BEM meshes comprised of up to 3000 and 6500 linear elements for the periodic and randomly distributed models, respectively. Fig. 4 illustrates a typical BEM discretisation used to represent a random-fibre composite undergoing transverse deformation, and Fig. 5 shows the local mesh refinements in regions where fibres come into close proximity with each other. 


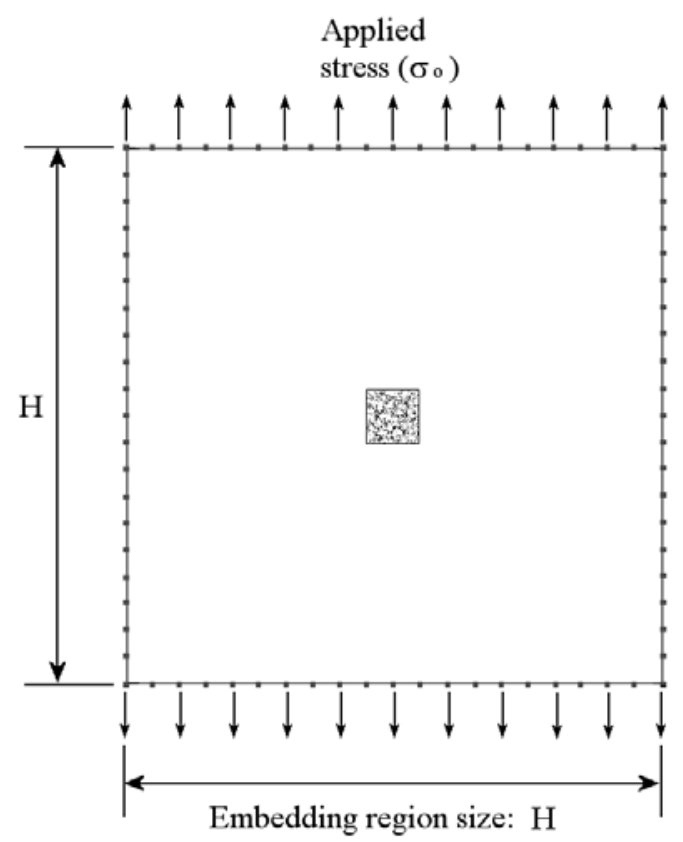

(a)

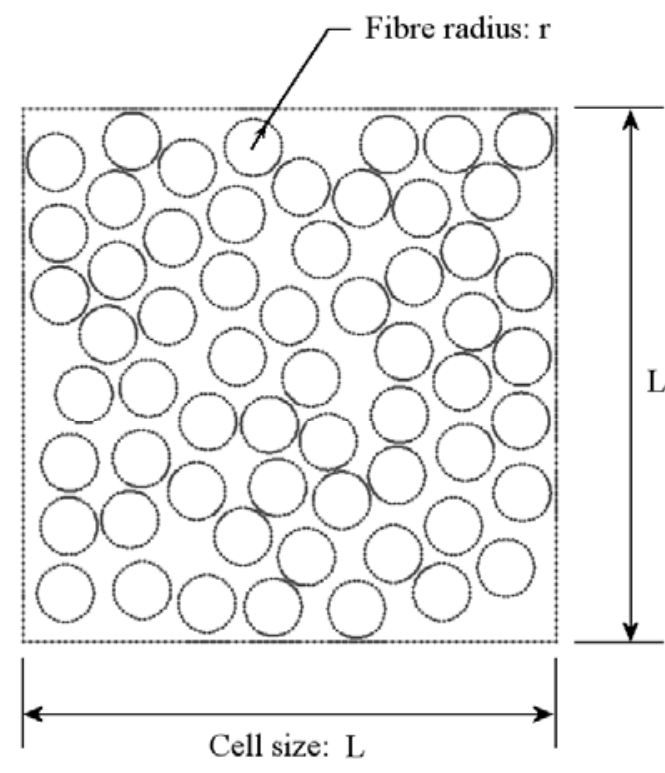

(b)

Figure 4 Typical 2D BEM model, showing (a) the full discretised model and (b) an enlarged view of the embedded cell. $(H / L=10.7, r / L=0.107)$

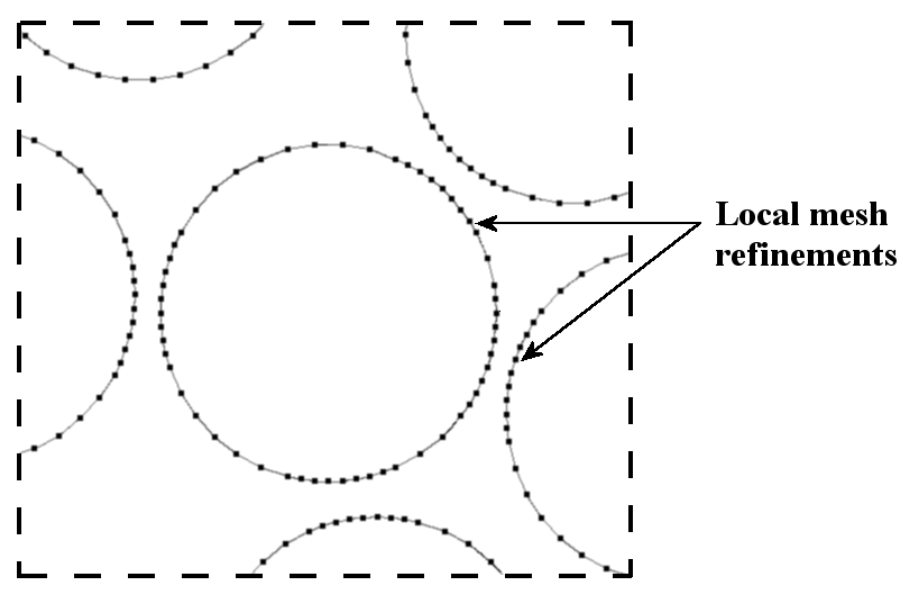

Figure 5 Close-up view BEM discretisation, indicating localised mesh refinements for near-touching boundaries. 
In the full model shown (Fig. 4), the upper and lower edges of the embedding region are subjected to a unit tensile stress $\left(\sigma_{o}\right)$ in the $y$ direction, and the model geometry is defined by: $H / L=10.7, r / L=0.107$, where $H, L$ and $r$ represent the embedding region size, cell size and the fibre radius, respectively. In addition, each fibre has the same radius, and the minimum ligament length is defined as ten percent of the fibre radius. The latter condition is stipulated such that the ligament size is of the same order of magnitude or greater than the elemental size in those regions. The reason for this restriction is to avoid the stresses becoming unbounded when the fibres touch, hence the problem to be considered has a contiguity $(C)$ value of zero.

\section{RESULTS AND DISCUSSION}

In this section, firstly embedded cell models containing regularly spaced periodic fibre arrangements are considered, followed by the more complex analysis involving embedded cells containing 60 randomly distributed fibres. The regular arrays are employed as benchmark studies to validate the modelling techniques and the numerical method. The random-fibre representations are used to investigate further the roles played by fibre distribution and constituent material properties on the localised response of 'real' fibre-reinforced materials.

\subsection{PERIODIC ARRANGEMENTS}

\subsubsection{Effect of cell size and spurious 'boundary layer'}

To quantify the 'boundary layer' produced by the ECA, a number of simulations were conducted using increasing size embedding cells (RVEs), which have a fibre volume fraction of 0.6. For the square (SQ) arrangement, normal $\left(T_{n}\right)$ and tangential $\left(T_{t}\right)$ tractions were taken around the central fibre within a single, nine, and twenty-five inclusion RVEs, as shown in Fig. 6(a). The corresponding results for a single, seven, and nineteen inclusion hexagonal (HEX) arrangements can be seen in Fig. 6(b). Note that, in all cases, the tractions have been normalized by the applied stress $\left(\sigma_{o}\right)$. 

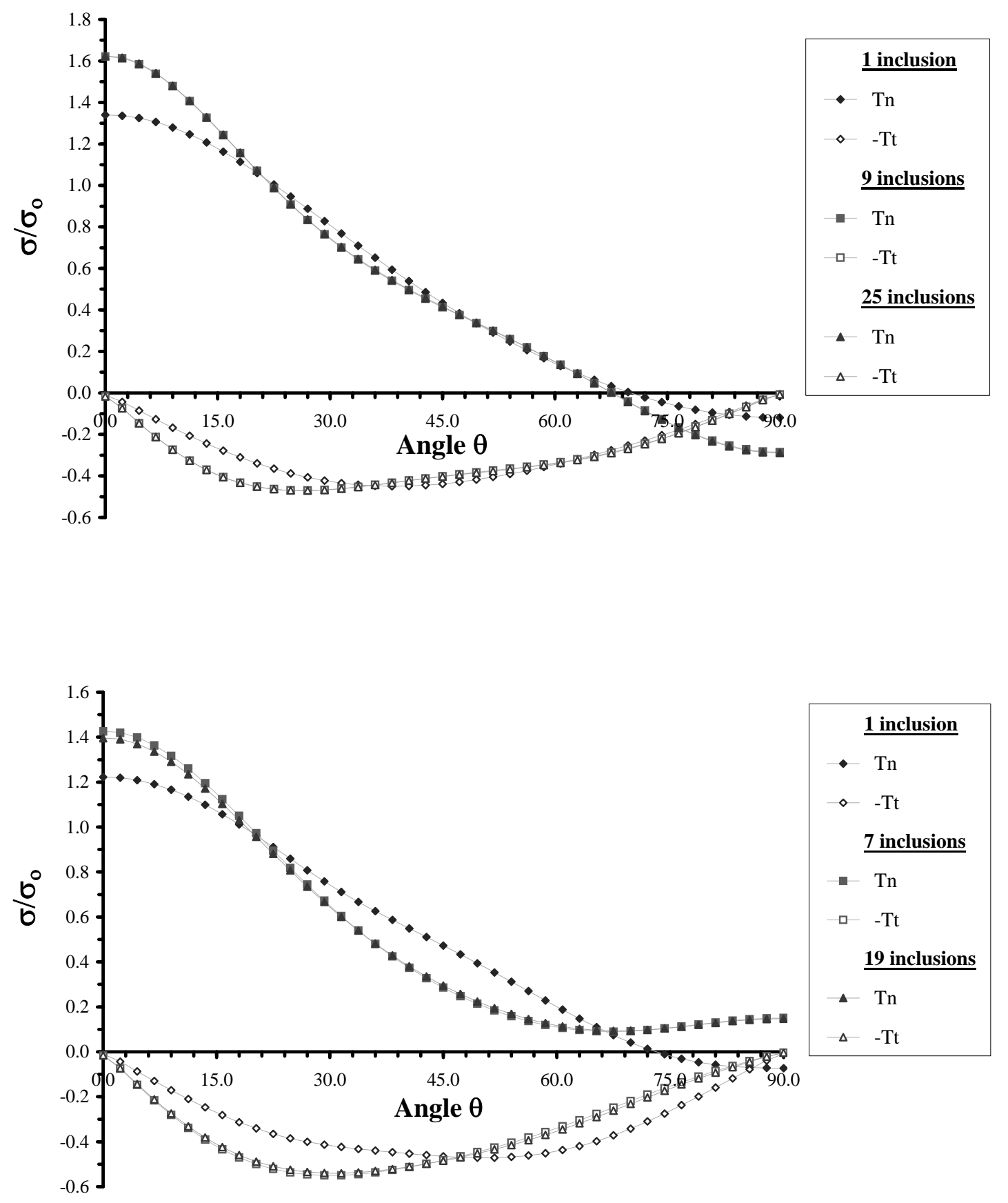

Figure 6 Effect of the periodic cell size on the normal and tangential interfacial tractions around the central inclusion: (a) Square arrangement; (b) Hexagonal arrangement. $\left(V_{f}=0.6, E_{f} / E_{m}=10, v_{f}=v_{m}=0.3\right)$

From these figures, it is noticeable that the distributions presented for the two larger embedded cells are very similar, however the values obtained from a single inclusion model are clearly dissimilar. This would suggest that, in both cases of square and 
hexagonal packing, the single inclusion model is insufficient in size to capture the localised behaviour, with the boundary layer introducing a noticeable effect. Nevertheless, for the resulting trends of the two larger embedded cells to be so similar, the boundary layer must be limited to a range of approximately one inclusion diameter, which is in agreement with literature (Böhm, 2000).

\subsubsection{Validation of the modelling techniques and the numerical method}

A comparison between the results presented for the largest embedded cells and the authors' FEM simulations using unit cell models with periodic boundary conditions, generally shows good agreement (see Fig.7). A range of volume fractions were analysed and the slight differences between the results have been found to be due to the approximated effective properties of the embedding region. As expected, the normal interfacial tractions $\left(T_{n}\right)$ are highest at $\theta=0^{\circ}$, the direction of the imposed tensile stress, and the magnitude of these tractions increases as the volume fraction rises, with values being slightly higher for the square packing than the hexagonal array. At $\theta=90^{\circ}$, the square-packed array predicts that the normal traction becomes compressive, whereas the hexagonal array remains tensile. The interfacial shear traction $\left(T_{t}\right)$ rises to a maximum for values of $\theta$ between $20^{\circ}$ and $45^{\circ}$ in both the square and hexagonal arrays.

Further studies considering periodic arrangements with different material properties were also undertaken, analysing the effect of varying the elastic moduli ratio $\left(E_{f} / E_{m}\right)$ and Poisson's ratio mismatch $\left(v_{f} / v_{m}\right)$ on the local stress concentrations. Discussion of these results, as well as a direct comparison with random arrangements, is given in section 3.2.3. 

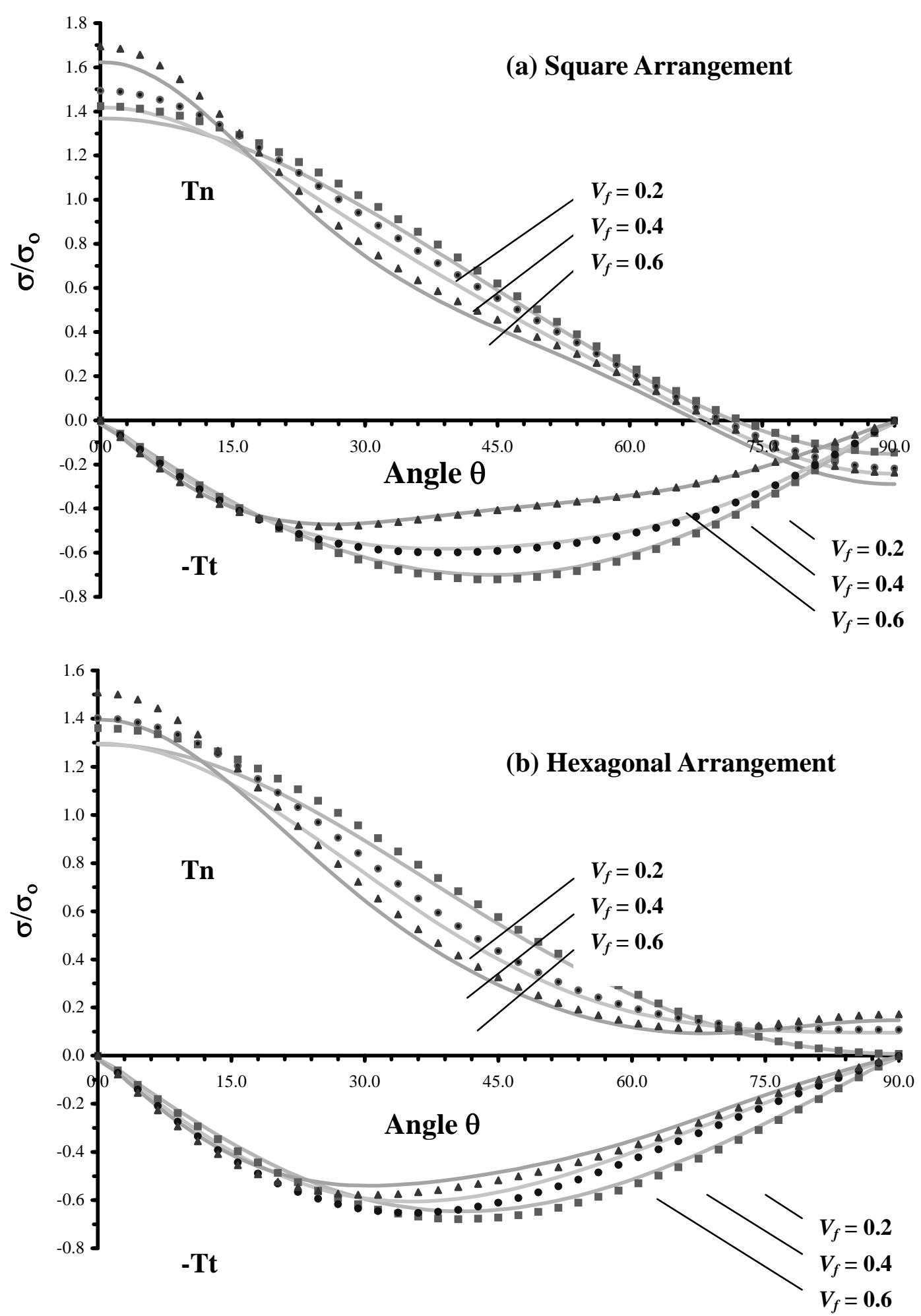

Figure 7 Comparison between BEM embedded cell models (symbols), and FEM unit cell models, with periodic boundary conditions (lines): (a) Square arrangement - 25 inclusions; (b) Hexagonal arrangement - 19 inclusions. $\left(E_{f} / E_{m}=10, v_{f}=v_{m}=0.3\right)$ 


\subsection{RANDOM ARRANGEMENTS}

\subsubsection{Geometric characterisation of the model}

As mentioned in section 2.2, the random-fibre embedded cell models were generated such that fibres do not overlap with the cell boundaries. Hence, the localised fibre volume fraction close to the cell walls would be expected to be less than that of the central region (or kernel). Therefore, to analyse the extent of this geometric variation, 'test' windows were placed within the embedded cell and the resulting sample's fibre volume fraction $\left(V_{f}\right)$ were numerically evaluated. The test window initially took the dimensions of the embedded cell, and the window boundaries were shifted inwards from the cell boundaries in steps of $0.1 r$, calculating $V_{f}$ at each step. The variation of $V_{f}$ within 10 random (RAND) embedding cells can be seen in Fig. 8. When the test window exactly matches the embedded cell, the average volume fraction of fibres is obtained. However, as the test window shrinks towards the central region (or kernel), the concentration of fibres increases in each model to a reasonably constant value at about one-inclusion diameter in from the cell walls. Although slightly deceptive in the figure due to its scaling, a consistency of the geometric characteristics is shown. The average kernel volume fraction $\left(V_{f} \approx 0.6\right)$ has a variation of less than $2 \%$, and all ten arrangements are within a 5\% scatter band.

From these statistical variations, and the observations found within the previous section regarding the size of the 'boundary layer' produced by the ECA, results taken within one-inclusion diameter from the cell walls are less representative. Hence, within subsequent analyses, results will only be taken within the embedded cell kernel. 


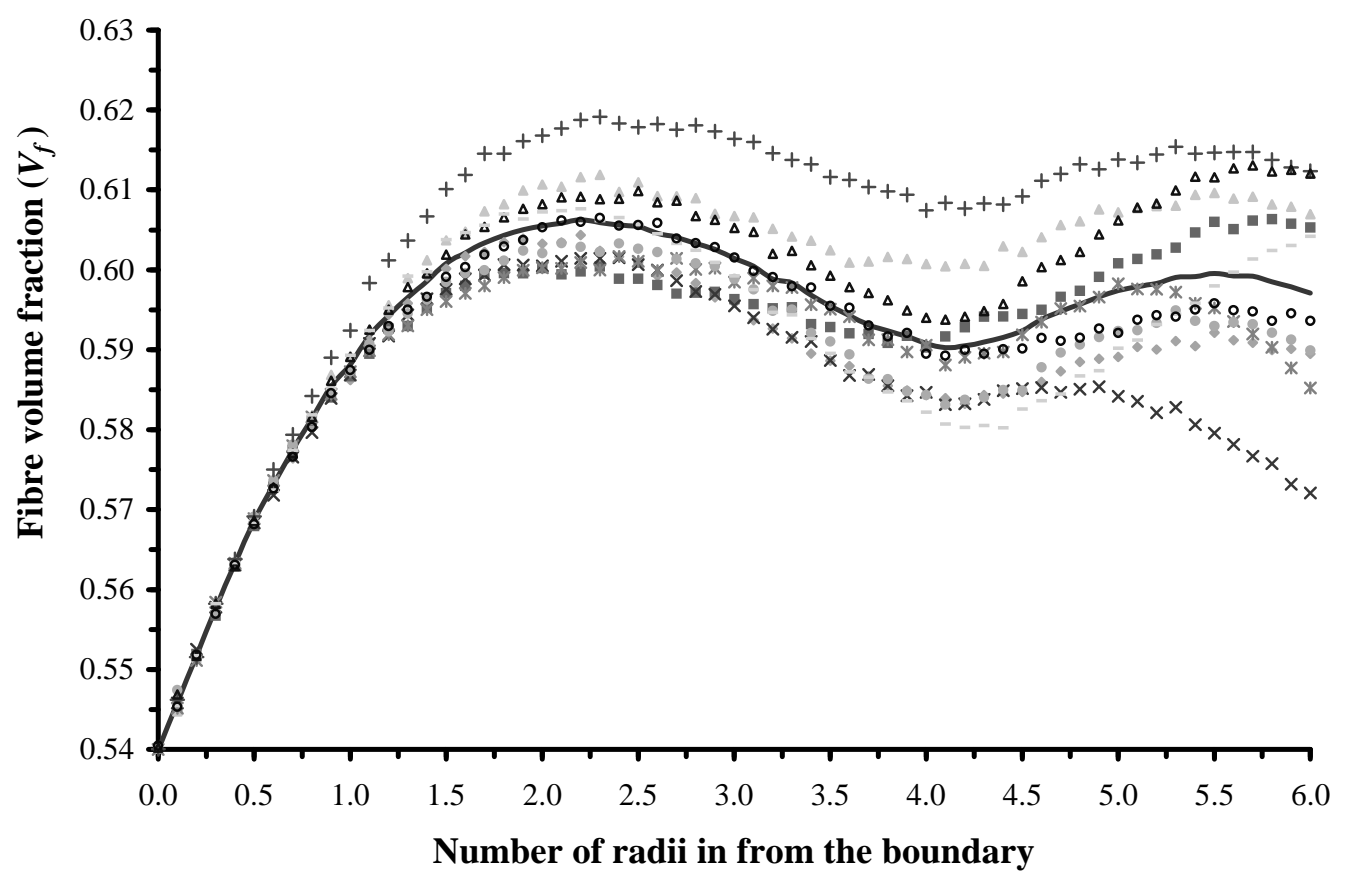

Figure 8 The variation in the fibre volume fraction $\left(V_{f}\right)$ within 10 random embedding cells. (Symbols indicate the individual geometric models analysed and the line shows the average variation)

\subsubsection{Local analysis of the composite}

Figure 9 shows a contour plot of the variation of the first principal stress (S1) within a typical random-fibre embedded cell model. Many interesting features are noticeable from this figure. For instance, tensile bands seem to be formed linking fibres which have centers aligned in a direction close to that of the applied load. This is clearly seen on the left-hand side of the figure, where two visible fibre chains (shaded in green through to red) are shown. This would indicate that the load is being transferred between neighbouring inclusions in such a way that direct load paths are formed, linking the upper and lower surfaces of the cell. Within these bands, high localised stresses can also be found at the fibre/matrix interface or within the matrix ligament, for inclusions that are nearly touching and are closely aligned with the applied stress. These stress concentrations are typically twice the applied stress, but can be even greater. 

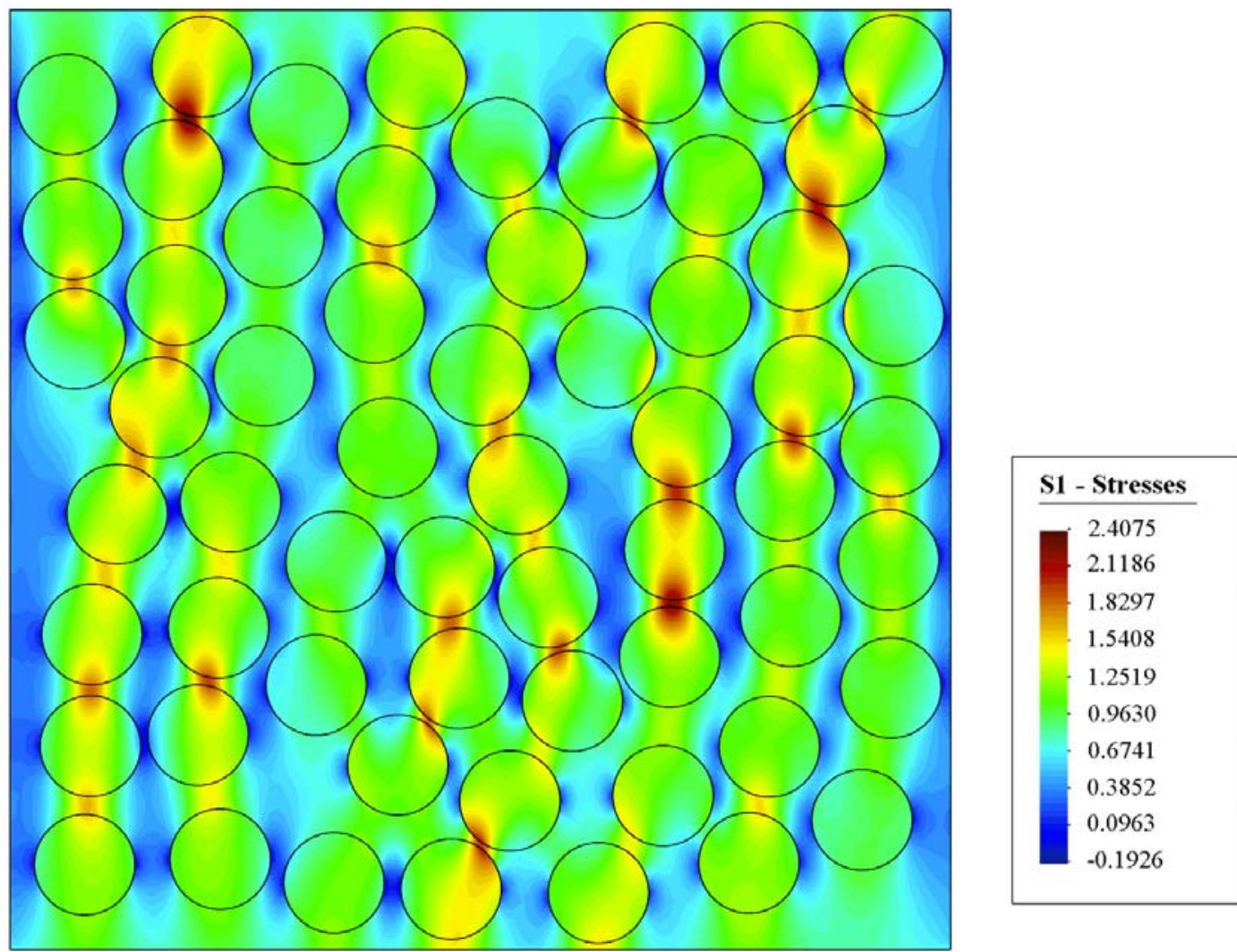

Figure 9 Contour plot of the variation of the first principal stress (S1) within a typical random-fibre embedded cell model. $\left(V_{f}=0.54, E_{f} / E_{m}=10, v_{f}=\right.$ $\left.v_{m}=0.3\right)$

For a periodic arrangement, consideration of the stress distribution within and around a single representative inclusion is sufficient to characterize the overall stress state. However, for the random arrays, the consideration of the stress distribution is not as straightforward, hence, alternative methods of considering and describing the results have to be used. As there is no standard conventional approach to this, new methods of general applicability are proposed, with the present results being used as particular examples.

From observations of the stress raiser locations and the local geometric arrangements of the fibres, it would seem that the magnitude of the stress concentrations are dependent on both the ligament length $(d)$ (distance between the two neighbouring fibres that have a common high-stress region, in units of $r$ ), and their angle ( $\delta$ ) relative to the applied 
load (angle between a plane containing the two fibre centres and the applied load). To investigate this phenomenon, maximum first principal stresses (S1) $\max$ were taken from each fibre and its surrounding matrix, within the kernel of ten random arrangements, as well as the corresponding values for $d$ and $\delta$. Figure 10 shows two scatter graphs, which relate to; (a) the results obtained within the fibres and (b) the results obtained from the surrounding matrix. In Fig. 10(a), the maximum fibre values were always at the interface between the inclusion and matrix, whereas, the maximum matrix values, Fig. 10(b), were either taken from the interface or within the ligament. In addition, the results tended to be slightly higher within the matrix, when compared to the corresponding values within the fibres.

Trends indicated within these figures seem to suggest that the magnitudes of the stress concentrations have a strong dependence on the ligament length $(d)$, and to a lesser extent on the angle $(\delta$ ) relative to the applied load. For example, data points that are distant from the two axes have lower values of stress than points close to the origin, and points of highest stress (shaded in red) are generally within a band $d=0.1-0.2 r$ and $\delta=$ $0-30^{\circ}$.

To examine the data shown in Fig. 10 in terms of statistical variations, the applicability of a Weibull analysis (Weibull, 1951) was considered. This distribution was chosen because it is generally used to analyse the failure statistics for brittle materials. A cumulative probability distribution was obtained by summing the number of stress results above a certain stress concentration factor $(K)$ and dividing this value by the total number of results in the set. This process was carried out over the range of stress concentrations, in intervals of 0.05 , as shown in Fig. 11. The triangular and diamond shaped symbols indicate the raw data corresponding to the matrix and fibres, respectively. 

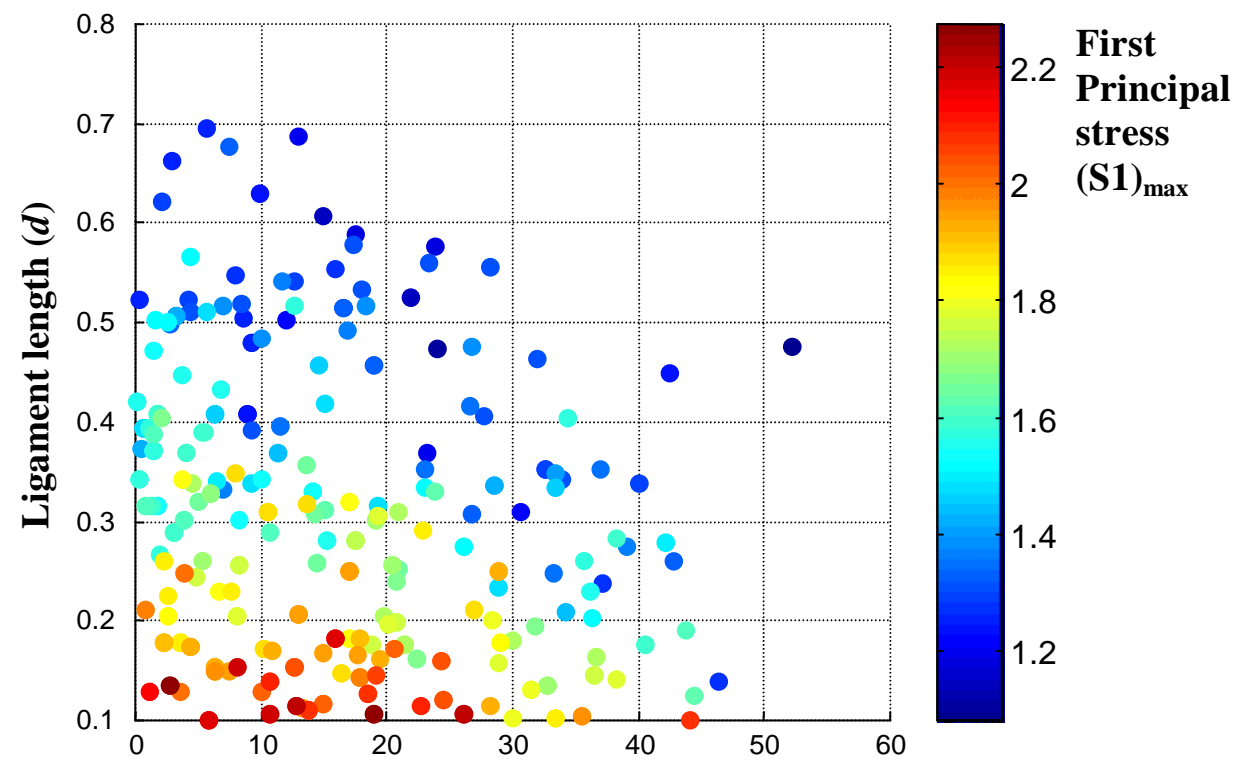

Angle relative to the applied load $(\delta)$

a)

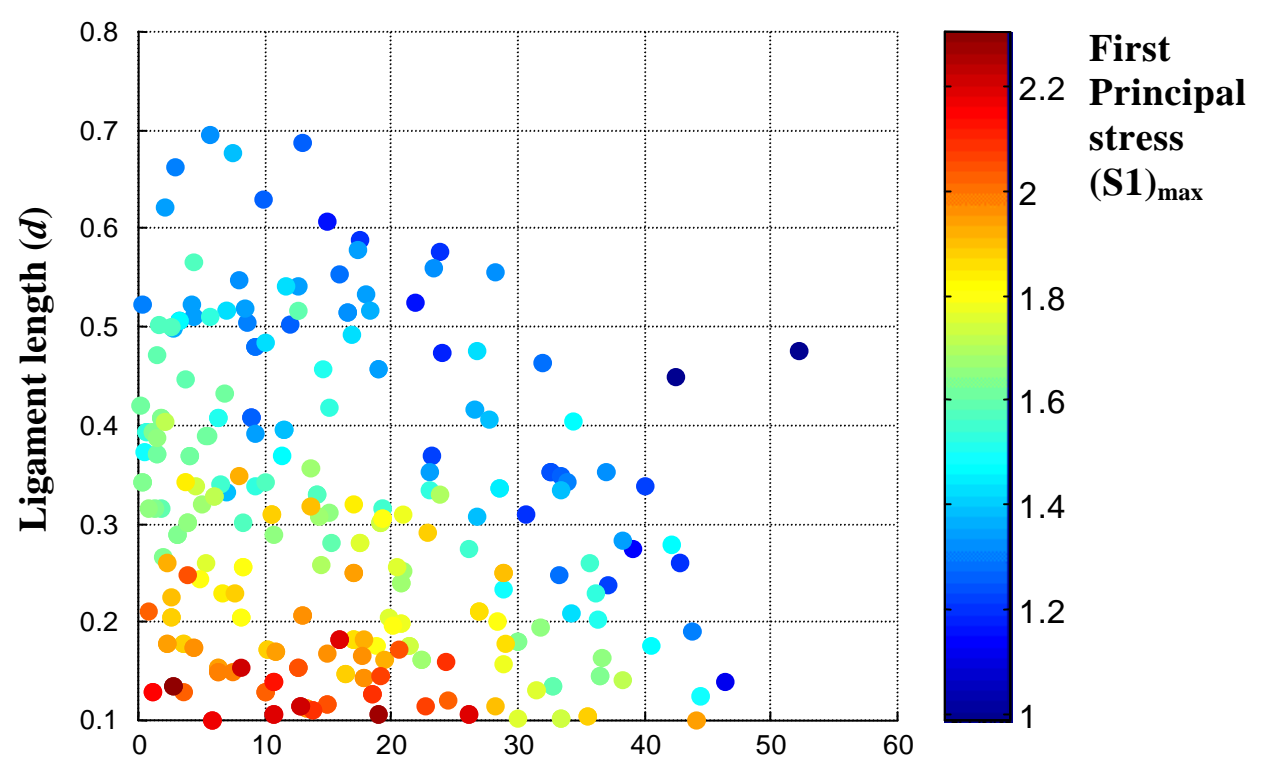

Angle relative to the applied load $(\delta)$

b)

Figure 10 Scatter graph showing the dependence of the localised maximum first principal stresses (S1) $\max$ within the 10 random arrangements on the ligament length and the alignment of the fibres relative to the applied load. Values are taken within (a) the fibres, and (b) the matrix. 
To obtain a curve fit to the data points, the standard three-parameter Weibull reliability function was employed, where the general form of the function $R(x)$ is given by:

$$
R(x)=\mathrm{e}^{-\left(\frac{x-\gamma}{\alpha}\right)^{\beta}}
$$

where $\alpha$ (scale parameter), $\beta$ (shape parameter) and $\gamma$ (location parameter) are positive constants.

To estimate the parameters that gave the 'best fit' to the data, a procedure involving linear transformation of the above equation, linear regression (to obtain $\alpha$ and $\beta$ ), and a simple iterative scheme to minimise any curvature within the transformed data (to obtain $\gamma$ ) was applied. As the results from the fibres and matrix are very similar (see Fig. 11) a single curve fit was made, using averaged values. From results shown in Fig. 11, it is first noticeable that the stress concentrations in the fibres and matrix are greater than unity, for all analysed cases. In addition, the shape parameter would seem to indicate that there is a reasonably high degree of variability in the stress concentration factors (taking a value of $\beta \leq 3$ as indicating high degree of scatter).

\subsubsection{Effect of the constituent elastic properties on the local behaviour}

To analyse whether the constituent material properties play a significant role on the local stress concentrations, further simulations were conducted using the values indicated in Table 1 . As mentioned previously, the elastic moduli ratio $\left(E_{f} / E_{m}\right)$ and Poisson's ratio mismatch $\left(v_{f} / v_{m}\right)$ of the phases will be isolated, and the maximum first principal stresses (S1) $\max$ retrieved from the different fibre packings. Two sets of results were obtained, corresponding to the fibres and matrix (in the same manner as those presented in Fig. 10). 


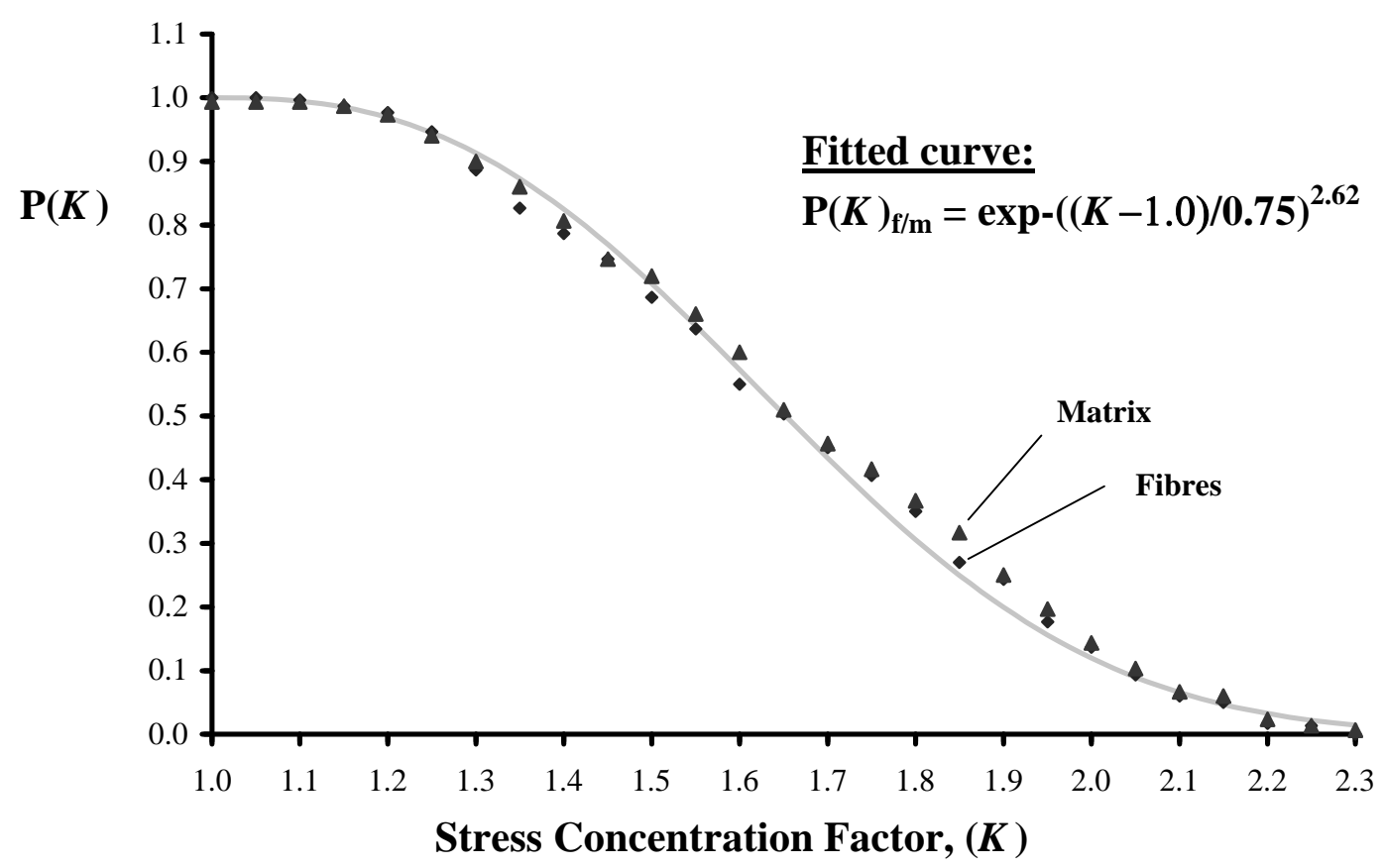

Figure 11 Cumulative Weibull distributions of the localised maximum first principal stresses $(\mathrm{S} 1)_{\max }$ within the fibres and matrix. $\left(V_{f}=0.54, E_{f} / E_{m}=10, v_{f}=\right.$ $\left.v_{m}=0.3\right)$

Considering first the effect of the elastic moduli, it is noticeable that for almost rigid fibres $\left(E_{f} / E_{m}=100\right)$ the maximum stress within the fibres and matrix increases, when compared to the previously analysed case $\left(E_{f} / E_{m}=10\right)$, as expected. This is prevalent in each of the geometric arrangements, although the effect is greatest for the random-fibre model ( 33\% increase), and then the square array ( 11\% increase), and finally the hexagonal array ( $\sim 2 \%$ increase). Next, considering a composite comprised of relatively compliant fibres $\left(E_{f} / E_{m}=0.1\right)$, the maximum stresses within the fibres decrease, whereas within the matrix values substantially increase. This is due to the fact that the matrix has to carry the majority of load, and also the transmission of this load is generally across very small ligament lengths.

By introducing a Poisson's ratio mismatch the local stress magnitudes are slightly affected, as shown in Table 1. If the Poisson's ratio of the matrix is higher than that of the fibres $\left(v_{f} / v_{m}=0.5\right)$, the stresses tended to be between $6-10 \%$ higher than the 
original model, where the Poisson's ratio of the phases were the same $\left(v_{f} / v_{m}=1\right)$. Whereas, if the Poisson's ratio of the matrix is lower than that of the fibres $\left(v_{f} / v_{m}=2\right)$, the stresses tended to be $2-7 \%$ lower than the original model.

\begin{tabular}{|c|c|c|c|}
\hline $\begin{array}{c}\text { Constituent } \\
\text { Material }\end{array}$ & $\begin{array}{c}\text { Square (SQ) } \\
\text { Arrangement } \\
\text { Properties }\end{array}$ & $\begin{array}{c}\text { Hexagonal (HEX) } \\
\text { Arrangement } \\
(25 \text { inclusions) }\end{array}$ & $\begin{array}{c}\text { Random (RAND) } \\
\text { Arrangement } \\
\text { (60 inclusions) }\end{array}$ \\
\hline$E_{f} / E_{m}=100$, & $1.855(\mathrm{~m})$ & $1.500(\mathrm{~m})$ & $3.060(\mathrm{~m})$ \\
$v_{f}=v_{m}=0.3$ & $1.800(\mathrm{f})$ & $1.426(\mathrm{f})$ & $3.012(\mathrm{f})$ \\
\hline$E_{f} / E_{m}=10$, & $1.675(\mathrm{~m})$ & $1.469(\mathrm{~m})$ & $2.305(\mathrm{~m})$ \\
$v_{f}=v_{m}=0.3$ & $1.625(\mathrm{f})$ & $1.397(\mathrm{f})$ & $2.273(\mathrm{f})$ \\
\hline$E_{f} / E_{m}=0.1$, & $4.906(\mathrm{~m})$ & $5.047(\mathrm{~m})$ & $7.084(\mathrm{~m})$ \\
$v_{f}=v_{m}=0.3$ & $0.518(\mathrm{f})$ & $0.537(\mathrm{f})$ & $0.916(\mathrm{f})$ \\
\hline$E_{f} / E_{m}=10$, & $1.798(\mathrm{~m})$ & $1.684(\mathrm{~m})$ & $2.537(\mathrm{~m})$ \\
$v_{f}=0.2, v_{m}=0.4$ & $1.719(\mathrm{f})$ & $1.564(\mathrm{f})$ & $2.486(\mathrm{f})$ \\
\hline$E_{f} / E_{m}=10$, & $1.618(\mathrm{~m})$ & $1.369(\mathrm{~m})$ & $2.231(\mathrm{~m})$ \\
$v_{f}=0.4, v_{m}=0.2$ & $1.590(\mathrm{f})$ & $1.329(\mathrm{f})$ & $2.215(\mathrm{f})$ \\
\hline
\end{tabular}

Table 1 The maximum first principal stresses (S1) $\max$ within models consisting of different fibre arrangements and constituent material properties. (Two sets of results are indicated, where results are taken within the fibres (f), and matrix (m), respectively.)

\subsubsection{Analysis of a composite with a stiff and compliant fibre mixture}

Finally, composite models comprising of 50:50 mixtures of both stiff and compliant fibres relative to that of the matrix are analysed. Figure 12 shows a contour plot of the variation of the first principal stress (S1) within a typical random-fibre embedded cell model with mixed inclusion properties. Comparing Fig. 9 (contour plot from a model whose geometric characteristics are the same, however, all the fibres have the same 
material properties) with Fig. 12, significant differences in the stress distribution are noticeable. In particular, the compliant inclusions are all virtually unstressed, whereas the stiff inclusions are generally experiencing stresses in excess of those present in previous models. This would indicate that the higher moduli inclusions are predominantly carrying the load, and as there are fewer stiff fibres the stresses within each would increase. Furthermore, very high-localised stresses can be found in the ligaments between compliant fibres that are generally aligned perpendicular to the applied load plane. In the particular model shown, the local stress concentrations are over six times higher than the applied stress. High stresses in these regions would be due to the transmission of local forces across relatively narrow cross-sections, in a similar manner to the results presented in section 3.2.3 for compliant inclusions.
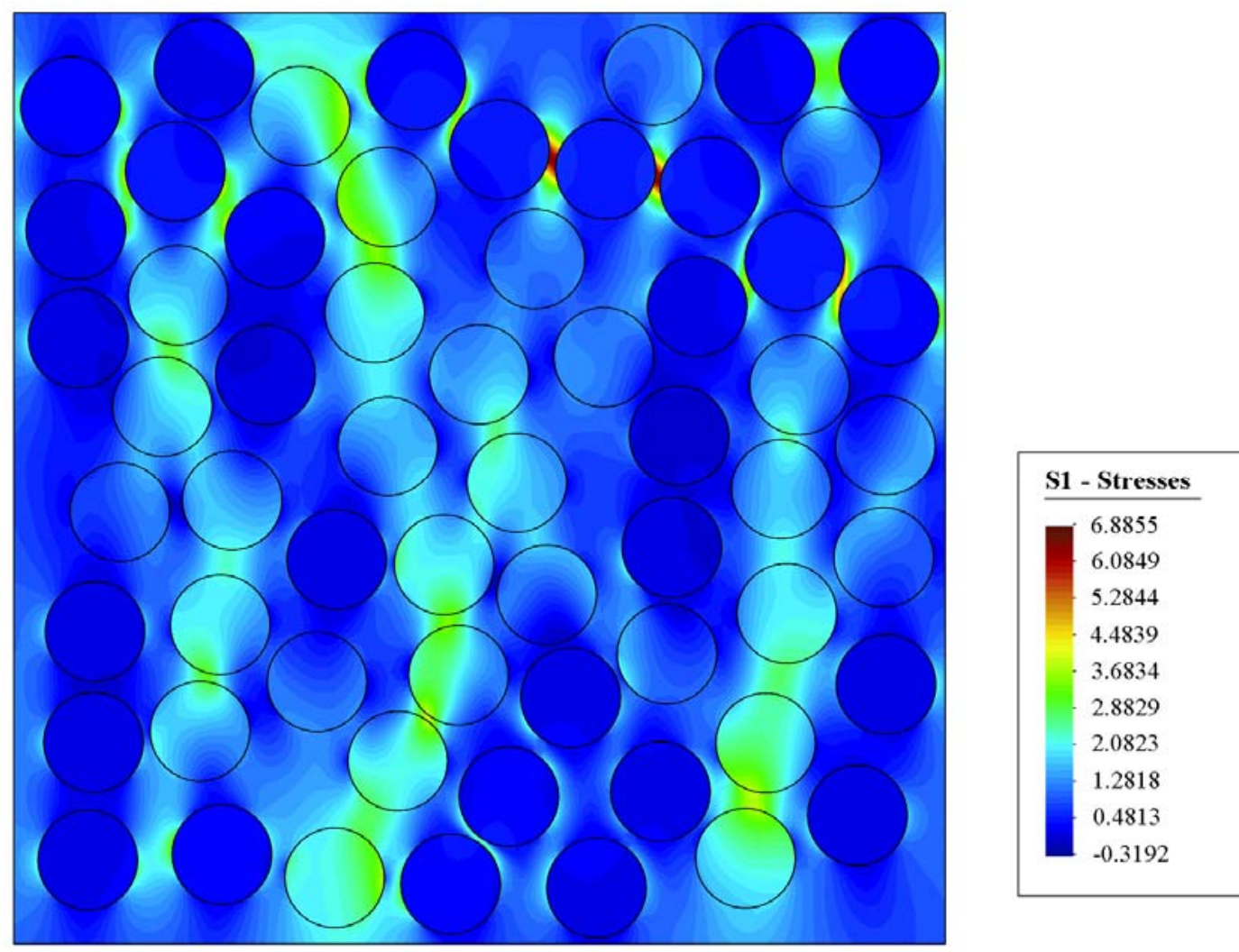

Figure 12 Contour plot indicating the variation of first principal stress (S1) within a typical random-fibre embedded cell model. $\left(V_{f}=0.54,50 \%\right.$ fibres $-E_{f} /$ $E_{m}=10,50 \%$ fibres $-E_{f} / E_{m}=0.1, v_{f}=v_{m}=0.3$ ) 
Statistical analysis of this three-phase system, once again, took the form of a Weibull distribution. Ten different arrangements were examined and in each case the geometric characteristics were kept constant, however the distribution of fibre property was randomly varied. Note that the fibre stresses were only taken from the stiff inclusions, as the stresses within compliant inclusions were less than the applied load. A similar Weibull plot to that of the two-phase system (Fig. 11) can be seen in Fig. 13 for the aforementioned three-phase system. Note that in this figure the fibre and matrix distributions are distinctly dissimilar, hence two separate curve fits were conducted.

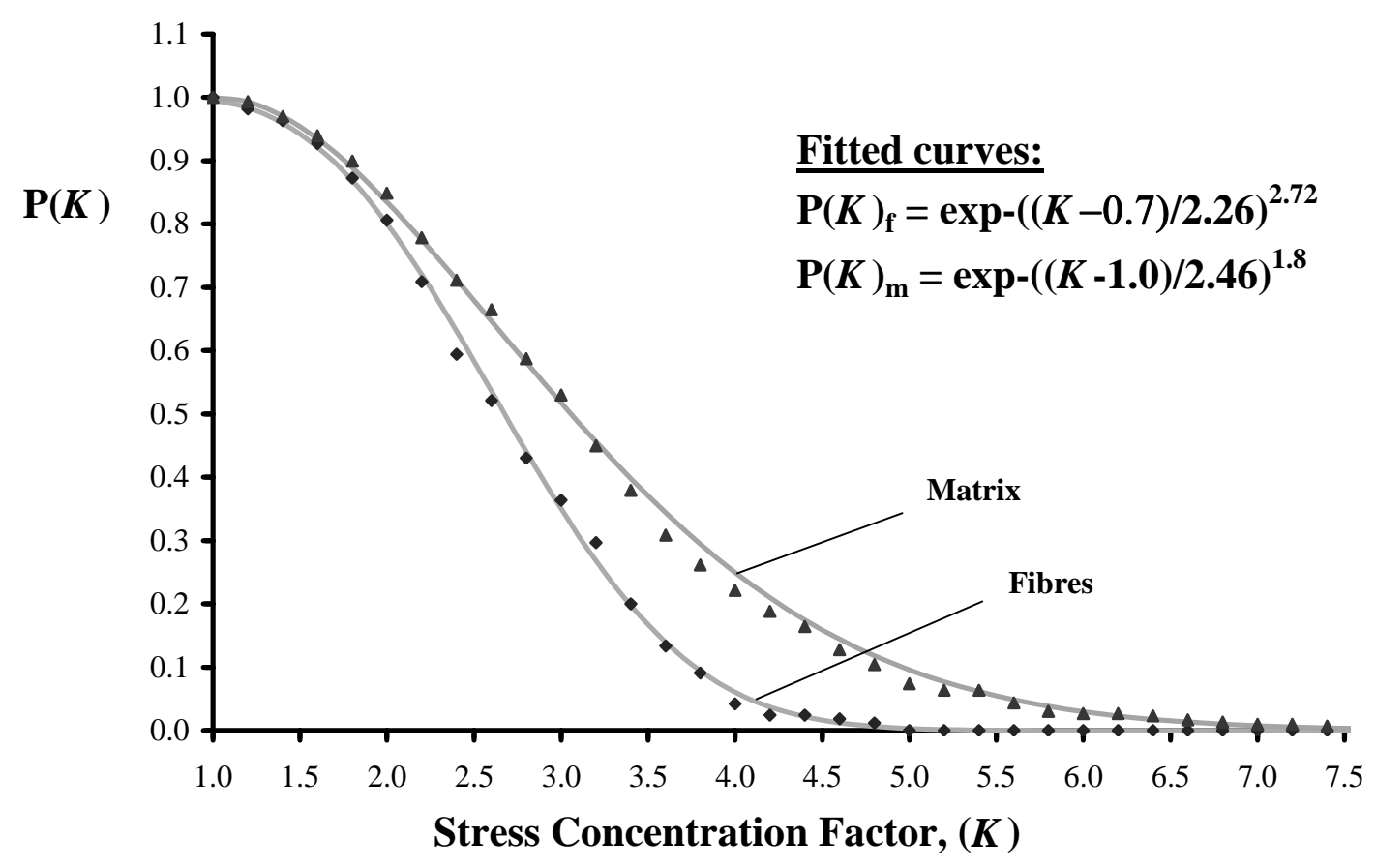

Figure 13 Cumulative Weibull distributions of the localised maximum first principal stresses (S1) $)_{\max }$ within the fibres and matrix. $\left(V_{f}=0.54,50 \%\right.$ fibres $-E_{f} /$ $E_{m}=10,50 \%$ fibres $-E_{f} / E_{m}=0.1, v_{f}=v_{m}=0.3$ )

Comparing figures 11 and 13, significant differences in the Weibull distributions are noticeable. In particular, the stress concentrations are substantially higher in the case of the three-phase system. This is indicated by the large difference in the scale parameters $\alpha$, used in the curve fits ( $\alpha=2.26$ and 2.46 for stiff fibres and matrix, respectively, in the three-phase system, and $\alpha=0.75$ for both the fibres and matrix of the two-phase 
system). The shape parameter $\beta$ would seem to indicate that there is a reasonably high degree of variability in all the results, and in particular, matrix stresses from the mixed-moduli model show a very high degree of variability $(\beta=1.8)$. Finally, the location parameter $\gamma$ shows that for the two-phase system, and the matrix of the three-phase system, the stress concentration factors (for the analysed cases at least) are always greater than one. However, for the stiff fibres of the mixed-moduli models, there is a higher likelihood of a stress reduction.

For a number of stress concentrations factors, i.e. $K=2,4$ and 6 , the corresponding probabilities can be calculated using the fitted Weibull functions, as shown in Table 2. This highlights the possible adverse effect, in terms of high localised stress concentrations, of mixing both stiff and compliant fibres relative to that of the matrix within a composite. Micro-cracks, which often nucleate in and around the embedded phase, would have a higher probability of initiation in the three-phase system, which in turn could lower the inherent strength of the composite.

\begin{tabular}{|c|c|c|c|}
\hline \multirow{2}{*}{$\begin{array}{c}\text { Stress } \\
\text { Concentration } \\
\text { Factor, } K\end{array}$} & $\begin{array}{c}|c| \\
\text { Pwo-phase, } \\
\text { fibres and matrix }\end{array}$ & $\begin{array}{c}\text { Three-phase, } \\
\text { stiff fibres }\end{array}$ & $\begin{array}{c}\text { Three-phase, } \\
\text { matrix }\end{array}$ \\
\cline { 2 - 4 } 2.0 & $1.19 \times 10^{-1}$ & $8.01 \times 10^{-1}$ & $8.21 \times 10^{-1}$ \\
\hline 4.0 & $3.87 \times 10^{-17}$ & $6.08 \times 10^{-2}$ & $2.39 \times 10^{-1}$ \\
\hline 6.0 & $2.64 \times 10^{-63}$ & $3.87 \times 10^{-5}$ & $2.77 \times 10^{-2}$ \\
\hline
\end{tabular}

Table 2 Calculated probabilities of the two-phase and three-phase systems having a stress concentration factor $(K)$ of 2,4 , and 6 . 


\section{SUMMARY}

In this study, the localised stress distributions within a multi-fibre composite undergoing transverse deformation are investigated using computational techniques. A combination of the boundary element method (BEM) and an embedded cell approach (ECA) allows a number of idealised (periodic) and more practical (random) discrete phase arrangements to be analysed in depth. Results obtained are for purely elastic material combinations and calculated stresses are given within the fibres, matrix, and fibre/matrix interfacial zone (assuming no debonding between the fibres and matrix).

The main results obtained from this study are:

- Fibre packing arrangement has a significant effect on the overall stress distribution and the magnitude of localised stress concentrations. For the same fibre volume fraction significantly higher stresses were observed in the non-periodic, compared to the periodic square and hexagonal arrangements.

- Constituent material properties also control the local response. For an elastic moduli ratio greater than unity, stress concentrations were present in the ligaments of near-touching inclusions that have centers aligned in a direction close to that of the applied load. The magnitudes of these stresses increased as the elastic moduli ratio increased. For a system of compliant fibres, very high stresses were present in the ligament of near-touching inclusions that have centers aligned in a perpendicular direction to that of the applied load. Finally, a Poisson's ratio mismatch only slightly affected the local stress distributions and their magnitudes.

- For the random arrangements of stiff fibres, the magnitudes of the stress concentrations seem to have a strong dependence on the ligament length $(d)$, and to a lesser extent on the angle relative to the applied load $(\delta)$.

- By mixing a compliant third-phase material into the composite, local stress concentrations substantially increased, which could lead to a reduction in the 
materials overall strength.

\section{ACKNOWLEDGEMENTS}

This investigation was supported by the Engineering and Physical Sciences Research Council (EPSRC).

\section{REFERENCES}

Aboudi, J. (1989), Micromechanical analysis of composites by the method of cells, Appl. Mech. Rev., 42, 193-221.

Achenbach, J. D. and Zhu, H. (1989), Effect of interfacial zone on mechanical behaviour and failure of fibre-reinforced composites, J. Mech. Phys. Sol., 37, 381-393.

Adams, D. F. and Doner, D. R. (1967), Transverse normal loading of a unidirectional composite, J. Comp. Mater., 1, 152-164.

Becker, A. A. (1992), The Boundary Element Method in Engineering - A Complete Course, McGraw-Hill, London.

Böhm, H. J. (2000), A short introduction to basic aspects of continuum micromechanics, Course notes: Frontiers of computational micromechanics in industry and engineering, European Advanced Summer School, Galway, Ireland.

Brebbia, C. A., Telles, J. C. F. and Wrobel, L. C. (1984), Boundary Element Techniques - Theory and Applications in Engineering, Springer-Verlag, Berlin.

Budiansky, B. (1965), On the elastic moduli of some heterogeneous materials, J. Mech. Phys. Solids, 13, 223-227.

Chati, M. K. and Mitra, A. K. (1998), Prediction of elastic properties of fiber-reinforced unidirectional composites, Engineering Analysis with Boundary Elements, 21, 235-244.

Chen, H. R., Yang, Q. S. and Williams, F. W. (1994), A self-consistent finite element approach to the inclusion problem, Comput. Mater. Sci., 2, 301-307. 
Christensen, R. M. (1990), A critical evaluation for a class of micro-mechanics models, J. Mech. Phys. Solids, 38, 379-404.

Dong, M. and Schmauder, S. (1996), Modelling of metal matrix composites by a self-consistent embedded cell model, Acta Mater., 44, 2465-2478.

Drugan, J. and Willis, J. R. (1996), A micromechanics-based nonlocal constitutive equation and estimates of representative volume element size for elastic composites, J. Mech. Phys. Sol., 44, 497-524.

Eischen, J. W. and Torquato, S. (1993), Determining elastic behaviour of composites by the boundary element method, J. Appl. Physics, 74, 159-170.

Foye, R. L. (1966), An evaluation of various engineering estimates of the transverse properties of unidirectional composites, Proceedings of the $10^{\text {th }}$ National SAMPE Symposium -Advanced Fibrous Reinforced Composites.

Guild, F. J. and Young, R. J. (1989), A predictive model for particulate-filled composite materials: Part 1 - Hard particles, J. Mater. Sci., 24, 298-306.

Gusev, A. A. (1997), Representative volume element size for elastic composites: A numerical study, J. Mech. Phys. Sol., 45, 1449-1459.

Halpin, J. C. and Tsai, S. W. (1967), Environmental factors in composite design, Air Force Materials Laboratory Technical Report, AFML-TR-67-423.

Hashin, Z. (1962), The elastic moduli of heterogeneous materials, J. Appl. Mech., 29, 143-150.

Hashin, Z. and Rosen, B. W. (1964), The elastic moduli of fiber-reinforced materials, J. Appl. Mech., 31, 223-232.

Hashin, Z. and Shtrikman, S. (1963), A variational approach to the theory of the elastic behaviour of multiphase materials, J. Mech. Phys. Solids, 11, 127-140.

Hill, R. (1965), A self-consistent mechanics of composite materials, J. Mech. Phys. Solids, 13, 213-222. 
Hornsby, P. R. and Premphet, K. (1997), Fracture toughness of multiphase polypropylene composites containing rubbery and particulate inclusions, J. Mat. Sci., 32, 4767-4775.

Hornsby, P. R. and Premphet, K. (1998), Influence of phase microstructure on the mechanical properties of ternary phase polypropylene composites, J. Appl. Polym. Sci., 70, 587-597.

Jones, R. M. (1999), Mechanics of Composite Materials, $2^{\text {nd }}$ Ed., Taylor \& Francis, Inc.

Knight, M. G., de Lacerda, L. A., Henshall, J. L. and Wrobel, L. C. (2002), A parametric study of inclusion interaction in particulate- and fibre-reinforced materials using the boundary element technique, Journal of Strain Analysis, 37, 47-58.

Monaghan, J. and Brazil, D. (1997), Modeling the sub-surface damage associated with the machining of a particle reinforced MMC, Comput. Mater. Sci., 9, 99-107.

Mori, T. and Tanaka, K. (1973), Average stress in matrix and average elastics energy of materials with misfitting inclusions, Acta Metall., 21, 571-574.

Nakamura, T. and Suresh, S. (1993), Effects of thermal residual stresses and fibre packing on deformation of metal-matrix composites, Acta Metall. Mater., 41, 1665-1681.

Saad, Y. and Schultz, M. (1986), GMRES: A generalized minimal residual algorithm for solving nonsymmetric linear systems, SIAM J. Sci. Statist. Comput., 7, 856-869.

Sautter, M., Dietrich, C., Poech, M. H., Schmauder, S. and Fischmeister, H. (1993), Finite element modelling of a transverse-loaded fibre composite: Effects of section size and net density, Comput. Mater. Sci., 1, 225-233.

Walpole, L. J. (1966), On the bounds for the overall elastic moduli of inhomogeneous systems - Part I, J. Mech. Phys. Solids, 14, 151-162.

Weibull, W. (1951), A statistical distribution function of wide applicability, J. Appl. Mech., 18, 293-297. 
Willis, J. R. (1977), Bounds and self-consistent estimates for the overall properties of anisotropic composites, J. Mech. Phys. Solids, 25, 185-202.

Wolfenden, A. and Wolla, J. M. (1989), Mechanical damping and dynamic modulus measurements in alumina and tungsten fibre reinforced aluminium, J. Mater. Sci., 24, 3205-3212.

Wrobel, L. C. and Aliabadi, M. H. (2002), The Boundary Element Method, John Wiley \& Sons, Chichester.

Wulf, J., Steinkopff, T. and Fischmeister, H. (1996), FE-simulation of crack paths in the real microstructure of an Al(6061)/SiC composite, Acta Mater., 44, 1765-1779. 